\title{
Comparison of Postural Control Ability according to the Various Video Contents during Action Observations
}

\author{
Bon Wook Goo'1, Mi Young Lee ${ }^{2}$
}

'Department of Medical Science, Graduate School, Daegu Haany University, Daegu, Republic of Korea; ${ }^{2}$ Department of Physical Therapy, College of biomedical Science, Daegu Haany University, Daegu, Republic of Korea

Purpose: This study examined the effects of the type of video contents used for action observations on the ability to control posture.

Methods: The participants were 48 healthy adults. The two hands of the participants were crossed on both shoulders, and the other foot was placed in a straight line in front of the target to allow them to watch a video of the monitor. The videos were presented in random order with three video contents (natural, stable balance posture, and unstable balance posture) consisting of 30 seconds each. A 15-second resting time was given between each video. During action observation using various video content forms, the posture control ability was measured using a TekScan MetScan ${ }^{\circledR}$ system.

Results: The results revealed statistically significant differences in the area of movement and the distance by COP and distance by the type of action-observation videos, and the distance by the anteroposterior and mediolateral sides $(p<0.05)$. The stable balance posture and unstable balance posture video showed significant differences in the distance by the COP, anteroposterior, and mediolateral distance. $(\mathrm{p}<0.05)$

Conclusion: This study suggests that choosing the contents of the videos is important during action-observation training, and actionobservation training can help improve postural control.

Keywords: Action observation, Balance, Mirror neuron system, Posture control

\section{서 론}

자세조절은 정적 자세를 취하거나 동적 활동 동안 안정성(stability)과 정위(orientation)의 조절을 위해 균형을 유지하거나 조절하는 능력이 며, 정상적인 일상생활을 위해 필수적인 요소이다. ${ }^{1-3}$ 세부적으로 정 적 자세에서의 자세조절은 자세 안정성(postural steadiness)이라고 불 리며, 동요(perturbations)에 대한 동적 자세 반응은 자세 안정성(postural stability)이라고 한다. 이러한 자세조절 능력은 대상자가 정적 기 립 동안 발생하는 압력 중심(center of pressure, $\mathrm{COP}$ )의 미묘한 변화를 모니터링함으로써 분석되거나,5,6 서 있는 대상자에게 동요를 적용하 고 동요에 대한 대상자의 반응을 분석함으로써 측정 되어진다.-9 한 편, 자세조절 능력은 노화와 뇌졸중, 파킨슨병, 소뇌질환 등과 같은 다양한 신경학적 질환 등에 의해 부정적인 영향을 받는다. ${ }^{3}$ 손상된 자세조절의 증상은 신체 흔들림, 감각 및 반응 처리의 지연, 동요에 대 한 감소되고 경직된 반응이 있다. ${ }^{10}$ 결과적으로 손상된 자세조절은
일상생활에 영향을 주어 기능적인 독립을 어렵게 하고 이동과 관련 된 활동에서 낙상의 위험성을 증가시킨다. ${ }^{3}$ 이러한 문제점을 해결하 기 위해 임상에서는 균형 훈련, 시각적 피드백 훈련, 테이핑 요법, 로 봇 치료, 체간 안정화 운동, 거울 치료 등의 다양한 자세조절 및 균형 에 대한 중재가 이루어지고 있다.1-16

최근 선행연구에서는 신경학적 손상이 있는 환자를 대상으로 운 동치료에 인지적 접근을 추가한 동작관찰훈련(action observation training, AOT)이 운동 기능, 보행 및 균형 등의 기능 회복에 효과가 있 음을 보고하고 있다.1718 세부적으로, $\mathrm{Fu}$ 등 19 은 뇌졸중 환자에서 동작 관찰훈련을 통해 상지 운동 기능과 일상생활 활동의 능력 향상을 보 고하였다. Patrizio 등 ${ }^{20}$ 의 연구에서는 행동 관찰이 아급성기 뇌졸중 환자에서 손의 운동 기능을 향상시킬 수 있음을 확인하였다. 또한 Pelson 등21은 동작관찰훈련이 파킨슨병 환자의 보행 능력과 균형 향 상에 긍정적인 영향을 미친다는 것을 보고하였다.

이러한 훈련은 특정 동작을 관찰하고 모방하는 것으로 동작관찰
Received Jan 12, 2021 Revised Feb 15, 2021

Accepted Feb 24, 2021

Corresponding author Mi Young Lee

E-mail mykawai@hanmail.net
Copylight ( 2021 The Korean Society of Physical Therapy

This is an Open Access article distribute under the terms of the Creative Commons Attribution Non-commercial License (https:// creativecommons.org/license/by-nc/4.0.) which permits unrestricted non-commercial use, distribution, and reproduction in any medium, provided the original work is properly cited. 
훈련에서 운동학습 과정의 촉진은 거울신경 시스템의 활성화를 통 해 이루어진다.22 거울신경세포의 특징은 단순한 동작 보다 목표 관 련 동작을 관찰할 때 더 활성화된다. 23,24 또한 사진으로 제공되는 정 적인 동작보다는 동적인 동작을 관찰할 때 더 활성화되며, 동작의 목 표를 제시한 경우 더 많이 활성화된다..$^{25}$ 이처럼 동작관찰훈련의 적 용 시 효과적인 중재 효과를 위해 거울신경 시스템의 특징을 파악하 고, 제공되는 영상의 콘텐츠의 적절한 선택이 중요할 것이다. 자세조 절 능력에 대한 동작관찰훈련의 효과에 대한 연구는 보고되고 있으 나, 제시되는 영상의 다양한 콘텐츠에 대해 자세조절 능력에 동시적 으로 미치는 영향을 확인한 연구는 드물다. 따라서 본 연구의 목적은 자세조절이 요구되는 동작 동안 제시되는 영상의 다양한 콘텐츠에 따른 자세조절 능력을 비교하고자 하였다.

\section{연구 방법}

\section{1. 연구대상}

본 연구는 건강한 20 대 성인 48 명(남 24명, 여 24명)이 참여하였다. 대 상자의 선정 기준은 1) 균형과 관련된 신경학적 결함이 없는 자, 2) 근 골격계 손상이 없는 자, 3) 전정 기관의 문제 혹은 어지러움이 없는 자 로 선정하였다. 본 연구는 헬싱키 선언(Declaration of Helsinki)에 따라 수행되었으며, 실험 전 모든 대상자들에게 실험 목적과 실험 방법 등 에 대한 자세한 설명 후, 연구 참여에 자발적으로 동의한 대상자만 실 험에 참여하였다.

\section{2. 측정도구}

다양한 형태의 영상 콘텐츠를 사용한 동작관찰 동안 자세조절 능력 의 측정을 위해 TekScan MatScansystem (TekScan Inc, South Boston,
MA, USA)을 사용하였다. 이 장비는 HR 매트, 커프, USB, 2-port hub로 구성되어 있으며, $\mathrm{HR}$ 매트는 $5.715 \mathrm{~mm}$ 두께에 8,488개의 센서로 구성 되어 있다. Research foot ver. 7.0 (TekScan Inc, South Boston, MA, USA) 와 The Sway Analysis Module (SAM ${ }^{\mathrm{TM}}$, TekScan lnc, South Boston, MA, USA) 소프트웨어를 사용하여 $30 \mathrm{frames} / \mathrm{sec}$ 로 30 초간 자료를 수집하 였고, 압력 중심(center of pressure, $\mathrm{COP}$ )의 이동 영역(area), 이동 거리 (distance), 앞뒤(antero-posterior, AP) 및 안가쪽(medio-lateral ML)의 이 동 거리를 측정 및 분석하였다.

\section{3. 실험 절차}

검사 방법으로 대상자의 양손은 양쪽 어깨에 교차하여 놓고 한 발 앞 에 다른 발을 일자로 두고 선 자세(tandem standing)에서 정면에 위치 한 모니터의 영상을 시청하도록 하였다. 각 30 초간의 영상을 시청하 는 동안 MatScan 장비를 이용하여 COP의 이동 영역, 이동 거리, 앞뒤 와 안가쪽 이동거리를 측정한다. 동작관찰에 사용되는 동영상의 콘 텐츠는 총 3 가지로 각 30 초의 자연(natural)영상, 안정된 균형 자세(stable balance posture, SBP)영상, 불안정한 균형 자세(unstable balance posture, USBP)영상으로 구성되었다(Figure 1). 세부적으로 자연 영상 은 균형과 관련이 없는 자연 영상이고, 안정된 균형 자세 영상은 영상 에 나오는 대상자가 양손을 어깨 위에 교차하여 놓고 한 발 앞에 다 른 발을 일자로 두고 서 있는 자세로 30 초 동안 흔들림 없이 균형을 잘 잡고 서 있는 영상이며, 불안정한 균형 자세 영상은 영상에 나오는 대상자가 안정된 자세 영상의 자세와 동일하지만, 30 초 동안 몸통의 흔들림이 지속적으로 나타나며 균형을 잘 유지하지 못하고 서 있는 영상이다. 자연 영상은 유튜브에서 크리에이티브 커먼스의 동영상을 사용하였으며, 안정된 그리고 불안정한 균형 자세 영상은 직접 촬영 하였다. 3 개의 동영상은 무작위 순서로 주어지며 각 동영상 시청 사이
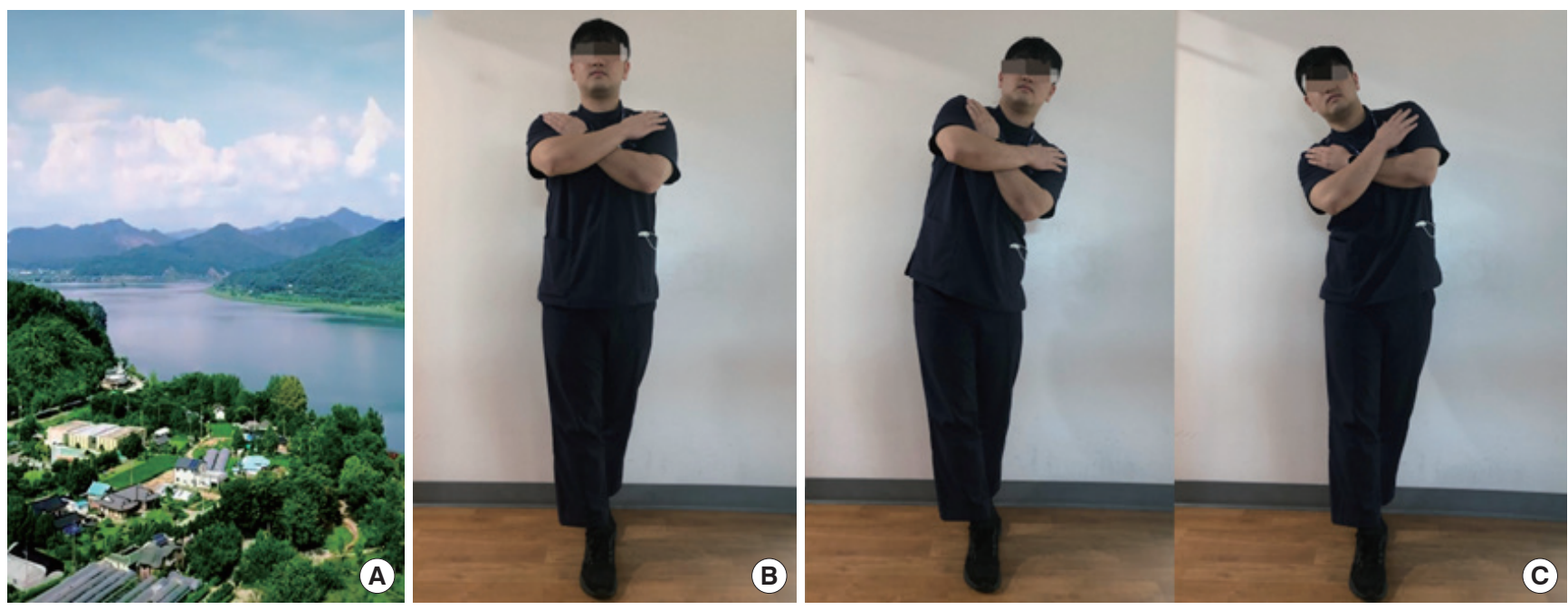

Figure 1. Video contents. A: Natural, B: SBP (Stable balance posture), C: USBP (Unstable balance posture). 
에는 15 초간의 휴식 시간이 주어졌다.

\section{4. 통계 방법}

본 연구의 모든 자료들은 SPSS (statistical package for the social science, Chicago, USA) Version 12.0을 사용하여 통계 분석을 수행하였다. 측정 된 값들은 Kolmogorov-Smirnov 검정을 사용하여 정규 분포를 확인 하였다. 동작관찰 영상의 종류에 따른 $\mathrm{COP}$ 의 이동 영역과 이동 거리 의 차이를 알아보기 위해 반복 측정 분산 분석(repeated ANOVA)과 대비검증(contrast test)을 사용하였다. 통계적 유의 수준은 $\mathrm{p}<0.05$ 로 설정하였다.

\section{결 과}

\section{1. 일반적 특성}

본 연구에 참여한 대상자는 남자 24 명, 여자 24 명으로 총 48 명이고, 평 균 연령 $24.1 \pm 1.4$ 세, 평균키 $166.8 \pm 8.1 \mathrm{~cm}$, 평균 몸무게 $64.8 \pm 16.3 \mathrm{~kg}$ 이 였다(Table 1).

\section{2. 동작관찰 영상의 종류에 따른 COP 이동 영역과 거리 비교}

동작관찰 영상의 종류(자연, 안정된 균형자세, 불안정한 균형 자세) 에 따른 $\mathrm{COP}$ 의 이동 영역과 이동 거리, 앞뒤와 안가쪽 이동 거리 모 두 통계적으로 유의한 차이를 보였다 $(\mathrm{p}<0.05)$. 대비검정에서 안정된 균형 자세 영상과 불안정한 균형 자세 영상은 $\mathrm{COP}$ 의 이동 거리, 앞뒤 와 안가쪽 이동 거리에서 유의한 차이를 보였다 $(\mathrm{p}<0.05)$. 그리고 안정 한 균형 자세 영상과 자연 영상은 $\mathrm{COP}$ 의 이동 영역과 이동 거리, 앞

Table 1. General characteristics of subjects

$(n=48)$

\begin{tabular}{lc}
\hline Variables & Subjects \\
\hline Gender (M/F) & $24 / 24$ \\
Age $(\mathrm{yr})$ & $24.1 \pm 1.4$ \\
Height $(\mathrm{cm})$ & $166.8 \pm 8.1$ \\
Weight $(\mathrm{kg})$ & $64.8 \pm 16.3$ \\
\hline
\end{tabular}

Mean \pm standard deviation.
뒤 이동 거리에서 유의한 차이를 보였다 $(\mathrm{p}<0.05)$. 불안정한 균형 자세 영상과 자연 영상은 유의한 차이가 없었다( $\mathrm{p}>0.05)$ (Table 2).

\section{고 찰}

본 연구에서는 동작관찰 동안 제시되는 영상의 다양한 콘텐츠가 자 세조절 능력에 미치는 영향을 알아보고자 하였다. 그 결과 영상의 콘 텐츠(자연, 안정된 균형 자세, 불안정한 균형 자세)에 따라 $\mathrm{COP}$ 의 이 동 영역과 이동 거리, 앞뒤와 안가쪽 이동거리 모두 통계적으로 유의 한 차이를 보였다. 특히 안정된 균형 자세 영상을 관찰할 때에는 다른 영상들에 비해 좋은 자세조절 능력을 보였으며, 불안정한 균형 자세 영상을 시청하였을 때에는 안정된 균형 자세의 영상보다 자세조절에 서 많은 흔들림이 보였지만 자연 영상과 유의한 차이가 없었다. 연구 결과는 영상의 콘텐츠에 따라 자세조절에 영향을 미쳤으며, 또한 대 상자는 콘텐츠와 유사한 자세조절 능력을 보였음을 확인하였다.

거울신경은 1996년도에 Giacomo Rizzolatti 등에 의해 원숭이 뇌의 앞운동영역(premotor area)에서 처음 발견되었으며, 원숭이가 물체를 집기와 같은 특정 손 동작을 수행할 때 같은 동작을 수행하거나 비슷 한 동작을 수행하는 다른 대상을 관찰할 때 활성화되는 신경원이 다. ${ }^{26}$ 인간에서 거울신경 시스템은 다른 사람의 행동을 반영하는 특 별한 신경원 그룹이며, 많은 뇌 영역에 의해서 형성되는 복잡한 신경 네트워크가 관여한다. 특히 핵심 거울신경 시스템으로 아래마루엽의 입쪽 부분(the rostal part of the IPL), 중심앞이랑의 아래 부분(lower part of the precentral gyrus)과 아래이마이랑의 뒤쪽부분(posterior part of inferior frontal gyrus) 영역이 있다. ${ }^{27}$ 거울신경 시스템의 기본적 인 기능은 관찰된 행동의 이해이며 또한 행동의 모방, 사회적 의사소 통과 감정, 사회 인지에 관여를 한다. ${ }^{28}$

한편, 동작관찰훈련이란 다른 사람이 수행하는 동작이나 영상 속 에 등장하는 인물의 행위에 대한 시각 및 청각 효과를 이용하여 관찰 자의 행동을 개선하기 위하여 사용되는 접근 방법이다. ${ }^{29}$ Buccino 등 30 은 동작관찰이 새로운 운동의 학습에 도움이 될 수 있으며, 관찰 대 상이 있을 때 거울신경 시스템이 활성화 된다고 보고하였다. 아울러,

Table 2. Comparison the of postural control ability according to various video contents

\begin{tabular}{|c|c|c|c|c|}
\hline & \multicolumn{3}{|c|}{ Video Content } & \multirow{2}{*}{$\mathrm{F}$} \\
\hline & Natural & Stable balance posture & Unstable balance posture & \\
\hline Area $\left(\mathrm{cm}^{2}\right)$ & $3.18 \pm 1.78$ & $2.45 \pm 1.24^{+}$ & $3.57 \pm 2.88$ & $7.555^{*}$ \\
\hline Distance (cm) & $77.00 \pm 15.03$ & $71.70 \pm 15.57^{+}$ & $75.12 \pm 16.40^{\ddagger}$ & $4.144^{*}$ \\
\hline AP Distance $(\mathrm{cm})$ & $2.72 \pm 0.75$ & $2.52 \pm 0.65^{+}$ & $2.94 \pm 0.97^{\neq}$ & $5.770^{*}$ \\
\hline ML Distance $(\mathrm{cm})$ & $2.77 \pm 0.81$ & $2.46 \pm 0.63$ & $2.93 \pm 0.95^{\neq}$ & 7.250 * \\
\hline
\end{tabular}

Mean \pm standard deviation, AP: antero-posterior, ML: medio-lateral.

${ }^{*} p<0.05$ : significant difference among three types of video contents.

${ }^{+} \mathrm{p}$ <0.05: significant difference between natural and stable posture videos.

${ }^{*} p<0.05$ : significant difference between stable and unstable posture videos. 
동작관찰과 신체훈련을 함께 시행하면 환자가 운동만 할 때 보다 재 활의 효율을 높여주며, 관찰된 행동을 실행하기 위해 거울신경 시스 템이 활성화되어 뇌졸중 후 운동 기능의 소실을 보이는 환자의 재활 에 긍정적인 영향을 미친다고 하였다. ${ }^{31}$ Ertelt 등 ${ }^{17}$ 은 동작관찰훈련 후 에 상지 기능의 향상을 확인하였고, 거울신경 시스템의 뇌활성도에 서도 차이가 났으며, 이것은 대상자들이 관찰된 동작을 운동 레퍼토 리에 일치시키고 모방하려는 과정에서 거울신경 시스템 영역의 활성 화를 가져왔다고 하였다. 이와 같이 거울신경 시스템의 기능과 동작 관찰에 대한 연구를 근거로 본 연구의 결과에서 안정된 균형 자세에 대한 영상을 관찰했을 때에는 불안정한 균형 자세에 대한 영상이나 자연 영상을 관찰했을 때 보다 좋은 자세조절 능력을 보였다.

결론적으로, 본 연구는 동작관찰 동안 제시되는 영상의 다양한 콘 텐츠에 따른 자세조절 능력을 비교해보았다. 연구 결과로 영상 콘텐 츠에 따라 자세조절 능력에 차이가 있었는데, 안정된 균형 자세 영상 을 관찰할 때에는 자연 영상과 불안정한 균형 자세 영상에 비해 좋은 자세조절 능력을 보여주었지만, 불안정한 균형 자세 영상을 관찰하 였을 때에는 자연 영상을 관찰할 때와 유사한 자세조절 능력을 보여 주었다. 즉, 자세조절을 필요로 하는 동작 동안에 제시되는 영상의 다 양한 콘텐츠 중 안정된 균형 자세 영상에서만 자세조절 능력에서 긍 정적인 영향을 받는 것으로 보이며, 불안정한 균형 자세 영상을 관찰 하는 것은 동작과 전혀 관련이 없는 자연 영상과 유사한 것을 보아 자 세조절 능력에 의미 있는 영향을 주지 않는 것으로 보인다. 본 연구는 제시된 동작을 관찰 또는 모방 후 자세조절 능력을 확인한 기존 연구 와는 다르게 자세조절을 필요로 하는 동작 동안 제시되는 영상의 다 양한 콘텐츠가 자세조절 능력에 동시적으로 미치는 영향을 비교했 다는 데 의의가 있다. 본 연구 결과를 근거로 임상에서 동작관찰훈련 의 효과를 높이기 위해 영상의 콘텐츠 선택과 구성이 중요하다는 것 을 시사한다. 본 연구의 제한점은 비교적 자세조절 능력이 좋은 20대 의 건강한 성인을 대상으로 하였기 때문에 균형 능력에 손상이 있는 환자에게서 동일한 결과를 예측하기가 어렵고 또한, 일회의 측정으 로 동작 관찰의 장기간 적용에 대한 효과를 알 수 없다는 것이다. 추 후 연구에서는 이러한 제한점들을 고려한 연구들이 지속적으로 이 루어져야할 것으로 생각된다.

\section{REFERENCES}

1. Pendergrass TL, Moore JH, Gerber JP. Postural control after a 2-mile run. Mil Med. 2003;168(11):896-903.

2. Pollock AS, Durward BR, Rowe PJ et al. What is balance? Clin Rehabil. 2000;14(4):402-6.

3. Shumway-Cook A, Woollacott MH. Motor control: translating research into clinical practice. Philadelphia, Lippincott Williams \& Wilkins, 2016:
4. Prieto TE, Myklebust JB, Myklebust B. Characterization and modeling of postural steadiness in the elderly: a review. IEEE Trans Rehabil Eng. 1993;1(1):26-34.

5. Winter DA, Patla AE, Prince F et al. Stiffness control of balance in quiet standing. J Neurophysiol. 1998;80(3):1211-21.

6. Winter DA, Prince F, Frank J et al. Unified theory regarding a/p and $\mathrm{m} / \mathrm{l}$ balance in quiet stance. J Neurophysiol. 1996;75(6):2334-43.

7. Gillette JC, Abbas JJ. Foot placement alters the mechanisms of postural control while standing and reaching. IEEE Trans Rehabil Eng. 2003;11 (4):377-85.

8. Henry SM, Fung J, Horak FB. Control of stance during lateral and anterior/posterior surface translations. IEEE Trans Rehabil Eng. 1998;6(1): $32-42$.

9. Nashner L, Cordo P. Relation of automatic postural responses and reaction-time voluntary movements of human leg muscles. Exp Brain Res. 1981;43(3-4):395-405.

10. Romero DH, Stelmach GE. Changes in postural control with aging and parkinson's disease. IEEE Eng Med Biol Soc. 2003;22(2):27-31.

11. Kim SH, Kim TH, Hwang HC. Effects of spinal stabilization exercises using visual feedback on gross motor function and balance in children with cerebral palsy. J Kor Phys Ther. 2019;31(5):279-85.

12. Son SM, Kang KW. Effect of action observation training using y-balance on balance capability in young adults. J Kor Phys Ther. 2020;32(2):65-9.

13. Song IY, Seo YS, Kang YH. Effects of 10-week body stability exercise program on functional movement and body balance of middle school volleyball players. J Kor Phys Ther. 2020;32(4):203-9.

14. Um YJ, Jang HY, Lee SM. Taping therapy simultaneously applied to the ankle and hip joint: effect on balance and gait in patients with chronic stroke. J Kor Phys Ther. 2019;31(1):49-55.

15. Yang H, Lim H. Effects of trunk control rehabilitation robot training on dynamic balance, lower extremity strength, gait ability and pain in bipolar hemiarthroplasty. J Kor Phys Ther. 2019;31(2):94-102.

16. Zeng W, Guo Y, Wu G et al. Mirror therapy for motor function of the upper extremity in patients with stroke: a meta-analysis. J Rehabil Med. 2018;50(1):8-15.

17. Ertelt D, Small S, Solodkin A et al. Action observation has a positive impact on rehabilitation of motor deficits after stroke. Neuroimage. 2007; 36:T164-T73.

18. Bhoraniya SH, Mishra DG, Parikh S. The effect of mirror therapy on the gait of chronic stroke patients: a randomized controlled trial. Natl J Physiol Pharm Pharmacol. 2018;8(9):1321-5.

19. Fu J, Zeng M, Shen F et al. Effects of action observation therapy on upper extremity function, daily activities and motion evoked potential in cerebral infarction patients. Medicine. 2017;96(42):e8080.

20. Sale P, Ceravolo MG, Franceschini M. Action observation therapy in the subacute phase promotes dexterity recovery in right-hemisphere stroke patients. Biomed Res Int. 2014;2014:457538.

21. Pelosin E, Barella R, Bet C et al. Effect of group-based rehabilitation combining action observation with physiotherapy on freezing of gait in parkinson's disease. Neural Plasticity. 2018;2018:4897276.

22. Rizzolatti G, Fogassi L, Gallese V. Neurophysiological mechanisms underlying the understanding and imitation of action. Nat Rev Neurosci. 2001;2(9):661-70.

23. Fogassi L, Ferrari PF, Gesierich B et al. Parietal lobe: From action organi- 
zation to intention understanding. Science. 2005;308(5722):662-7.

24. Rizzolatti G, Fadiga L, Gallese V et al. Premotor cortex and the recognition of motor actions. Cogn Brain Res. 1996;3(2):131-41.

25. Brass M, Bekkering H, Wohlschläger A et al. Compatibility between observed and executed finger movements: Comparing symbolic, spatial, and imitative cues. Brain Cogn. 2000;44(2):124-43.

26. Gallese V, Fadiga L, Fogassi L et al. Action recognition in the premotor cortex. Brain. 1996;119(2):593-609.

27. Rizzolatti G, Craighero L. The mirror-neuron system. Annu Rev Neurosci. 2004;27:169-92.
28. Rajmohan V, Mohandas E. Mirror neuron system. Indian J Psychiatry. 2007;49(1):66.

29. De Vries S, Mulder T. Motor imagery and stroke rehabilitation: a critical discussion. J Rehabil Med. 2007;39(1):5-13.

30. Buccino G, Riggio L. The role of the mirror neuron system in motor learning. Kinesiology. 2006;38(1):5-15.

31. Malouin F, Belleville S, Richards CL et al. Working memory and mental practice outcomes after stroke. Arch Phys Med Rehabil. 2004;85(2):17783. 